ORIGINAL ARTICLE

\title{
Secular trends of nocardia infection over 15 years in a tertiary care hospital
}

\author{
R Matulionyte, P Rohner, I Uçkay, D Lew, J Garbino
}

J Clin Pathol 2004;57:807-812. doi: 10.1136/jcp.2004.016923

See end of article for authors' affiliations

....................

Correspondence to: Dr J Garbino, Division of Infectious Diseases, Department of Internal Medicine, University Hospitals of Geneva, 24 rue Micheli-du-Crest, 1211 Geneva 14, Switzerland; Jorge.Garbino@hcuge.ch

Accepted for publication 16 February 2004
Aims: To assess the incidence of nocardia infection over 15 years in a tertiary care hospital.

Methods: Over a 15 year period, Nocardia spp were isolated from 20 patients hospitalised at the Geneva University Hospitals, Switzerland.

Results: Sixteen patients had one or more underlying conditions. The median time between symptom onset and diagnosis was 30 days. The most common initial unconfirmed diagnosis was pulmonary tuberculosis (four). The lung was involved in 16 cases, followed by the central nervous system (two) and skin (two); one patient had disseminated infection. The most common species identified was $N$ asteroides. In vitro susceptibility testing was performed on 14 of 20 strains. All strains were susceptible to imipenem and amikacin. Initial treatment with trimethoprim/sulfamethoxazole (TMP/SMX) was started in 14 patients, although five patients had to be switched to another treatment because of side effects or lack of efficacy. A cure was observed in 15 patients, death in three, and relapse or complications in two.

Conclusions: Nocardiosis can become a severe infection and mainly affects profoundly immunocompromised patients. Differential diagnosis often delays the time to diagnosis, which worsens the outcome. New diagnostic tools, such as the polymerase chain reaction, could provide more rapid and reliable results. TMT/SMX was the most commonly prescribed treatment, but needed to be changed for another treatment because of side effects or lack of efficacy in a considerable proportion of patients. Imipenem should be used as an alternative treatment for severely ill patients, and the sulfa combination for less severe infections.
$\mathrm{N}$ ocardiosis is a localised or disseminated infection caused by ubiquitous, soil borne, aerobic, and saprophytic actinomycetes. Although nocardia species can infect immunocompetent individuals, they most often affect immunocompromised patients. ${ }^{2}{ }^{2}$ Earlier reports estimated that 500 to 1000 cases/year occur in the USA, and probably 150 to 250 in France. ${ }^{34}$ The current incidence of nocardiosis in Europe is still unknown because of the lack of comprehensive reporting systems. ${ }^{5}$ Reports in the literature are limited to case reports and case series that include only a small number of patients. Moreover, nocardial infections can be difficult to recognise, which leads to misdiagnosis and consequently underestimation of its incidence..$^{5-7}$ For the same reason clinical experience remains limited.

"Nocardial infections can be difficult to recognise, which leads to misdiagnosis and consequently underestimation of its incidence"

In this study, we review the epidemiology, clinical, and microbiological characteristics, treatment, and outcome of nocardial infections diagnosed in 20 patients at the Geneva University Hospitals, Switzerland over a 15 year period.

\section{PATIENTS AND METHODS \\ Patients}

Our retrospective study was conducted at the 2200 bed Geneva University Hospitals, Switzerland. Cases of nocardiosis were identified with a computerised query of the clinical microbiology laboratory database for specimens collected between January 1989 and December 2003. Thereafter, the medical records for all patients with Nocardia spp were reviewed. Information on demographic characteristics, underlying conditions, immunosuppressive treatment, nocardiosis clinical, radiological manifestations and diagnostic methods, concomitant infections, treatment, and outcome were assessed.

\section{Definitions}

Nocardiosis was considered a definite diagnosis when Nocardia sp. was identified in a clinical specimen of a symptomatic patient. Disseminated nocardiosis was defined as nocardia infection in two or more non-contiguous sites and nocardaemia when Nocardia sp. could be isolated from blood cultures. Colonisation was defined as a positive culture of a specimen from a non-sterile site without clinical evidence of infection. Breakthrough nocardiosis was identified when a recurrent nocardial infection occurred in a patient receiving systemic antibacterials with known in vitro activity against Nocardia spp for at least four days before the onset of nocardial infection. Lymphopenia was defined as $<1000 \times 10^{6}$ lymphocytes/litre, neutropenia as $<500 \times 10^{6}$ neutrophils/litre, hypoproteinaemia as a serum concentration of protein $<60 \mathrm{~g} /$ litre, hypoalbuminaemia as a serum concentration of albumin $<35 \mathrm{~g} /$ litre, and raised lactate dehydrogenase $(\mathrm{LDH})$ as $>240 \mathrm{U} /$ litre.

Effective antimicrobial treatment was defined as when patients were cured or their clinical signs and symptoms of nocardiosis improved, including microbiological and radiographical findings. Failure was identified when patients died or deteriorated as a result of nocardial infection. Relapse was noted when an initial improvement was followed by the reappearance of clinical symptoms, radiographical findings, and the isolation of Nocardia sp.

Abbreviations: $B M T$, bone marrow transplantation; CNS, central nervous system; HIV, human immunodeficiency virus; LDH, lactate dehydrogenase; TMP/SMX, trimethoprim/sulfamethoxazole 


\section{Microbiological methods}

Nocardia spp were cultured on different media such as sheep blood agar (Columbia base), CNA, and chocolate agar incubated aerobically at $35^{\circ} \mathrm{C}$, in an atmosphere enriched with $5 \% \mathrm{CO}_{2} .{ }^{8}$ Identification was based on microscopical morphology after Gram stain and modified Kinyoun stain, on strictly aerobic growth, and on physiological tests (casein, tyrosine, xanthine, starch, gelatin, urea, susceptibility to lysozyme, and growth at $45^{\circ}$ ). Since 2001 , species identification has been determined by the amplification and sequencing of 16S rDNA. ${ }^{10}$ Susceptibility to antimicrobial agents was determined with E-test strips (AB Biodisk, Solna, Sweden) on Mueller-Hinton blood agar.

\section{RESULTS}

Over the 15 year study period Nocardia spp were isolated from 20 patients. In 16 patients, the infection was community acquired. The remaining four nosocomial cases were not clustered, because the patients developed the infection at different times and were hospitalised in different wards. Fourteen patients were male. The mean age was 51.8 years (range, 4-88).

Sixteen patients had at least one underlying condition responsible for a certain degree of immunodeficiency (table 1). One patient with chronic obstructive pulmonary disease was treated with longterm inhalational corticosteroids, and another 88 year old patient had a previous

Table 1 Nocardiosis characteristics in the 20 patients

\begin{tabular}{|c|c|}
\hline Characteristic & No of episodes (\%) \\
\hline \multicolumn{2}{|l|}{ Underlying condition } \\
\hline Solid organ malignancy* & $4(20)$ \\
\hline HIV infection & $3(15)$ \\
\hline Diabetes mellitus & $3(15)$ \\
\hline Solid organ transplantation $†$ & $3(15)$ \\
\hline Idiopathic lymphopenia & $1(5)$ \\
\hline Chronic granulomatous disease & $1(5)$ \\
\hline Common variable immunodeficiency & $1(5)$ \\
\hline \multicolumn{2}{|l|}{ Chronic lung disease } \\
\hline Cystic fibrosis & $1(5)$ \\
\hline Sarcoidosis & $1(5)$ \\
\hline $\begin{array}{l}\text { Corticosteroids administered one month before } \\
\text { nocardiosis } \ddagger\end{array}$ & $6(30)$ \\
\hline $\begin{array}{l}\text { Immunosuppressive chemotherapy administered } \\
\text { one month before nocardiosis }\end{array}$ & $4(20)$ \\
\hline Radiotherapy received during the previous year & $1(5)$ \\
\hline Coinfection & $4(20)$ \\
\hline \multicolumn{2}{|l|}{ Nocardia spp } \\
\hline$N$ asteroides & $13(65)$ \\
\hline$N$ asteroides complex & $4(20)$ \\
\hline$N$ farcinica & $1(5)$ \\
\hline N nova & $1(5)$ \\
\hline$N$ brasiliensis & $1(5)$ \\
\hline \multicolumn{2}{|l|}{ Site of infection } \\
\hline Pulmonary§ & $16(80)$ \\
\hline CNS & $2(10)$ \\
\hline Cutaneous & $2(10)$ \\
\hline Disseminated** & $1(5)$ \\
\hline \multicolumn{2}{|l|}{ Outcome } \\
\hline Cure/improvement & $15(75)$ \\
\hline Failure & $1(5)$ \\
\hline Relapse & $1(5)$ \\
\hline Death & $3(15)$ \\
\hline \multicolumn{2}{|c|}{ 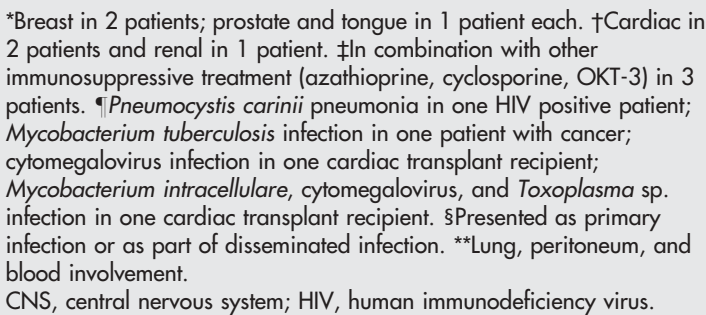 } \\
\hline
\end{tabular}

respiratory syncitial virus infection. No underlying disease could be identified in two children presenting with cutaneous nocardiosis. Pulmonary aspergillosis was diagnosed in two patients before nocardiosis; one of them had concomitant pulmonary tuberculosis and one a concomitant pulmonary paecilomyces infection. Breakthrough nocardiosis occurred in one patient with chronic granulomatous disease after prophylactic treatment with trimethoprim/sulfamethoxazole (TMP/SMX), 400/80 mg twice daily.

The median time interval between onset of symptoms and diagnosis was 30 days (mean, 24.9; range, 3-50 days). The most common initial diagnosis instead of nocardiosis was pulmonary tuberculosis in four patients, followed by bacterial sinusitis in two, and legionellosis, pulmonary embolism, and Wegener's disease in one patient each.

The most frequent abnormality identified by laboratory analysis was lymphopenia, which was seen in 15 patients. None of the patients had neutropenia. In three patients with human immunodeficiency virus infection, the CD4 counts ranged from 2 to $17 \times 10^{6}$ cells/litre. Raised LDH was seen in 10 of 11 patients, hypoproteinaemia in six of 13, and hypoalbuminaemia in eight of nine patients for whom laboratory results were available.

\section{Clinical characteristics}

The body site most frequently involved was the lung, followed by the skin and the brain. Disseminated infection was seen in one patient. Three patients with nocardaemia carried intravenous catheters. Two of them developed pulmonary nocardiosis and one developed disseminated nocardiosis.

All but one of the 16 patients with pulmonary involvement were symptomatic. Three patients presented an acute form of infection and 12 a subacute form. Three patients were hospitalised at the intensive care unit as a result of severe respiratory distress syndrome $(\mathrm{n}=1)$ or septic shock $(\mathrm{n}=2)$. Table 2 presents the clinical manifestations and radiographical features. Pulmonary nocardiosis was caused by $N$ asteroides sensu stricto in 12 patients, $N$ asteroides complex in 2, $N$ nova in one, and $N$ farcinica in one. Diagnostic cultures were obtained from at least one of the following clinical specimens: sputum in 10 patients, bronchoalveolar lavage in six, empyema drainage in three, and lung biopsy, abscess puncture fluid, and gastric fluid in one each. In 15 of 22

Table 2 Clinical manifestations and radiographical features in 16 patients with pulmonary nocardiosis

\begin{tabular}{ll}
\hline Finding & No (\%) of patients \\
\hline Clinical manifestations & $10(62.5)$ \\
Fever & $10(62.5)$ \\
Cough & $9(56.3)$ \\
Worsening asthenia & $8(50.0)$ \\
Weight loss & $3(18.8)$ \\
Pleuritic pain & $2(12.5)$ \\
Severe headache & $1(6.3)$ \\
Haemoptysis & \\
Radiographical features & \\
Infiltration & $5(31.3)$ \\
Lobar & $5(31.3)$ \\
Multilobar & $5(31.3)$ \\
Nodules & $2(12.5)$ \\
Multiples & $4(25.0)$ \\
Solitary & $3(18.8)$ \\
Pleural effusion & $3(18.8)$ \\
Empyema & $1(6.3)$ \\
Cavitation & $1(6.3)$ \\
Abscess &
\end{tabular}


pulmonary specimens Nocardia spp were visible on Gram stain.

The patient with disseminated nocardiosis as a result of $N$ asteroides had pneumonia with pleural effusion, perigastric abscess, peritonitis, and nocardaemia. The patient was a heart transplant recipient who had received prolonged immunosuppressive treatment. He was transferred to our hospital because of pneumonia of unknown aetiology, which had been treated with ciprofloxacin and clarithromycin for 21 days. At admission, he presented with sepsis, which rapidly progressed to septic shock as a result of concomitant cytomegalovirus infection.

Central nervous system (CNS) nocardiosis was diagnosed in the two patients with cerebral abscesses. The neurological features in one patient were aphasia, right central facial paresis, and right hemiparesis. The abscess was $3 \mathrm{~cm}$ in diameter in the left parietal anterior lobe. The second patient had an abscess of $2 \mathrm{~cm}$ diameter in the left cerebellum. He suffered from headache, nausea, and instability, and the computed tomography scan showed a solitary ring enhancing lesion with central necrosis and surrounding oedema within the corresponding localisation. Nocardial infection was limited to the CNS in both cases; Nocardia spp were suspected after examination of the Gram stain, and $N$ asteroides complex and $N$ asteroides sensu stricto grew from bacteriological cultures from the abscess specimens.

Nocardia brasiliensis and $N$ asteroides complex were identified in the cases of cutaneous nocardiosis.

\section{Treatment and outcome}

In vitro testing for the principal antibacterial agents was performed for 14 of 20 strains (table 3). Resistance to either imipenem or amikacin was not detected among the $N$ asteroides sensu stricto or $N$ asteroides complex strains. TMP/SMX and ceftriaxone showed high activity against the isolates, whereas ciprofloxacine, tobramycin, and gentamicin showed less anti-nocardial activity. The lowest activity against $N$ asteroides was noted for penicillin, amoxicillin, and amoxicillin-clavulanic acid.

Treatment targeting Nocardia spp was given to 17 of 19 patients (information was not available for one patient). Antibacterial agents with known anti-nocardial activity used in monotherapy or in combination included TMP/SMX in 14 patients, imipenem in seven, ciprofloxacin in four, and minocyclin, amikacin, and sulfadiazine in three each. The most common initial treatment consisted of TMP/SMX, oral
( $\mathrm{n}=7$ ) and parenteral $(\mathrm{n}=4)$. TMP/SMX had to be switched to another treatment because of side effects in three patients and because of a lack of efficacy in two other patients. The median treatment duration in surviving patients was 90 days (mean, 117; range, 10-360). In two patients with CNS involvement, a craniotomy with abscess evacuation was performed. Abscess debridement was carried out for the two patients with the cutaneous nocardiosis. For the patient with disseminated nocardiosis, a perigastric abscess was drained and a peritoneal rinse with drainage was performed.

A cure was obtained in 15 patients. One patient with chronic granulomatous disease experienced three relapses during the four month period after the pulmonary nocardiosis. The primary pulmonary infection and the relapse episodes occurred during prophylactic and curative treatment with TMP/SMX, and were caused by $N$ nova, which was susceptible in vitro to TMP/SMX. During the first episode, the patient was treated with parenteral TMP/SMX for three days, but was then switched to oral treatment. He was definitely cured after a six week treatment with imipenem in combination with amikacin for two weeks. A human immunodeficiency (HIV) positive patient with pulmonary nocardiosis who was treated with oral TMP/SMX had a cerebral infection one month later (identified radiologically as an appearance of multiple focal lesions in Virchov Robin and lenticular nucleus regions). Clinical improvement was achieved after a two week treatment with imipenem.

Death as a result of nocardial infection occurred in three patients. Among the patients who died, one patient with cancer had not been treated for the nocardia infection because the clinical manifestations had been attributed to tuberculosis. A second patient with cancer had been treated with oral TMP/SMX. In both patients, nocardia targeted treatment was introduced late-23 and 50 days after the onset of clinical manifestation, respectively. The third patient, a cardiac transplant recipient, was treated with oral sulfadiazine.

\section{DISCUSSION}

Previous reports indicate a possible increased incidence of nocardia infection. ${ }^{14}$ Our present study revealed that nocardiosis remains infrequent in our institution. The incidence over the 15 year study period stayed constantly low, with one to two new cases each year. The same findings have also been reported in other European countries. ${ }^{611}$

Table 3 Nocardia spp antimicrobial susceptibility

\begin{tabular}{|c|c|c|c|c|c|}
\hline \multirow[b]{2}{*}{ Antibiotic } & \multicolumn{5}{|c|}{ Susceptible strains/Tested strains } \\
\hline & $N$ asteroides & $\begin{array}{l}N \text { asteroides } \\
\text { complex }\end{array}$ & $N$ nova & $N$ farcinica & $N$ brasiliensis \\
\hline Penicillin & $0 / 3$ & - & - & - & - \\
\hline Amoxicillin & $1 / 7$ & - & - & - & - \\
\hline $\begin{array}{l}\text { Amoxicillin- } \\
\text { clavulanate }\end{array}$ & $1 / 4$ & - & - & - & - \\
\hline Piperacillin & $1 / 3$ & - & - & - & - \\
\hline Cefuroxime & $4 / 4$ & - & - & - & - \\
\hline Ceftriaxone & $6 / 6$ & - & - & $0 / 1$ & - \\
\hline Imipenem & $9 / 9$ & $2 / 2$ & $1 / 1$ & $1 / 1$ & $0 / 1$ \\
\hline TMP/SMX & $8 / 9$ & $2 / 2$ & $1 / 1$ & $1 / 1$ & $1 / 1$ \\
\hline Amikacin & $9 / 9$ & $2 / 2$ & - & $1 / 1$ & - \\
\hline Gentamicin & $4 / 6$ & - & - & $0 / 1$ & - \\
\hline Tobramycin & $2 / 3$ & - & - & - & - \\
\hline Ciprofloxacin & $5 / 8$ & - & - & $1 / 1$ & - \\
\hline Minocycline & $1 / 1$ & $2 / 2$ & - & - & - \\
\hline Tetracycline & $1 / 2$ & - & - & $0 / 1$ & - \\
\hline Vancomycin & $1 / 2$ & - & - & - & - \\
\hline Erythromycin & $0 / 3$ & - & - & - & - \\
\hline
\end{tabular}


Most patients were male, similar to most of the published reports. ${ }^{611-16}$ The reason for this distribution is unclear and may be related to hormonal effects on the virulence or growth of nocardia. ${ }^{17}$ However, the predominance in men shifted to equal proportions in the groups of patients with cancer $^{18}$ or lung transplant recipients. ${ }^{19}$

Although rare, nocardial infection can occur in immunocompetent patients. A study, in which 253 cases of nocardiosis were reviewed, found no evidence of underlying illness or immunosuppressive treatment in $15 \%$ of patients, ${ }^{3}$ and this figure ranges from $10 \%$ to $25 \%$ in other reports. $^{161217}$ In general, Most patients presenting with nocardiosis have a certain degree of immune deficiency. Activated macrophages and $\mathrm{T}$ cells constitute the major defence mechanism for nocardial infection, whereas B cells and humoral immunity do not appear as important in protecting the host. ${ }^{2}$ An incidence of $2.3 \%$ of nocardial infection was found in renal transplant recipients, ${ }^{20} 0.06 \%$ in patients with cancer, ${ }^{18} 0.3 \%$ in bone marrow transplant (BMT) recipients, ${ }^{13}$ and $0.38-1.8 \%$ in HIV positive patients. ${ }^{7121}$ Impaired local pulmonary defences seen in chronic obstructive pulmonary disease or other chronic pulmonary diseases predispose to pulmonary nocardiosis, particularly in patients requiring longterm corticosteroid treatment. ${ }^{15}$ Systemic immunosuppression predisposes to invasive pulmonary and disseminated infections, which is often the case in organ transplant recipients. ${ }^{15} 1922$ Colagenous vascular diseases, chronic granulomatous diseases, dysgammaglobulinaemia, alcoholism, and diabetes mellitus all enhance susceptibility to nocardiosis. Although some reports have indicated a surprisingly low incidence of nocardiosis among HIV infected patients, nocardiosis remains an important cause of morbidity and mortality in HIV positive patients with advanced infection, particularly in those not receiving TMP/SMX prophylaxis. ${ }^{71} 23$

\section{"The most common site of infection was the lung, which was involved in 13 patients"}

In our present study, 16 of the 20 the patients had at least one known underlying condition responsible for immunodeficiency. In addition, two patients had other predisposing factors such as longterm corticosteroid inhalation and transient immunosuppression secondary to respiratory syncitial virus infection. The most common underlying disease was solid organ malignancy, followed by solid organ transplantation, diabetes mellitus, and HIV infection. Surprisingly, none of our patients had a haematological malignancy or was a BMT recipient. Lymphocytopenia was seen in 15 of the 20 the patients, and the HIV positive patients were in a late stage of the disease. None of the patients in our study was neutropenic. Elsewhere, neutropenia was reported in only $10 \%$ of patients with cancer who had nocardiosis, ${ }^{18}$ and was not considered to be a significant risk factor in BMT recipients. ${ }^{13}$

The most common site of infection was the lung, which was involved in 13 patients; the subacute lung form, which is known as the typical manifestation of nocardiosis, ${ }^{5}$ was particularly prevalent. However, acute forms are common, especially in severely compromised hosts, ${ }^{18}$ and this form seems to be correlated with a poor prognosis. ${ }^{17}$ In our present study, all three patients with nocardaemia had central venous catheters. Such endovascular devices and prosthetic valves appear to be a main risk factor associated with bacteraemia. ${ }^{17}$ One of our patients with cystic fibrosis was colonised with $N$ asteroids. This confirms previous reports that the isolation of Nocardia spp from patients with cystic fibrosis does not necessarily imply active infection, but treatment is recommended to protect against potential dissemination. ${ }^{24}$
CNS involvement may be present in up to $20 \%$ of patients with nocardiosis. ${ }^{61-15}$ The two patients who we identified survived after neurosurgery in conjunction with antibiotic treatment. Nocardial brain abscesses can be life threatening and may cause diagnostic and therapeutic difficulties, particularly in patients with cancer. ${ }^{18}{ }^{25} \mathrm{~A}$ high mortality rate was reported in a Swiss study of cerebral nocardiosis as a result of underlying diseases or complications. ${ }^{25}$ A lower mortality rate was published in an Australian report, but only two of the 11 patients in that study were severely immunocompromised. ${ }^{26}$ Disseminated infection was uncommon in our present study, as reported by others, ${ }^{18} 21$ although in some other studies the prevalence was reported to be higher. ${ }^{3}{ }^{12} 14$ The cutaneous form of nocardiosis may be underdiagnosed in our region because Gram stain and cultures are not routinely performed for superficial skin lesions.

Clinical recognition of nocardial infection is difficult because of its relatively low incidence and a lack of pathognomic symptoms.. The chronic debilitating course of this infection often mimics tuberculosis, pneumocystosis, invasive fungal disease, or malignancy. ${ }^{20} 27$ Pleomorphic and non-specific radiological manifestations further complicate the diagnosis. In our present study, the median time between the onset of symptoms and diagnosis was long, with a mean of 30 days, a delay also reported elsewhere. ${ }^{21} 27$ The most common initial diagnosis instead of nocardiosis was pulmonary tuberculosis, which was suspected in four patients in our present study; this is because of the clinical similarity of these infections, particularly in HIV positive patients. ${ }^{21}{ }^{28-30} \mathrm{In}$ one case, Wegener's granulomatosis had been misdiagnosed when pulmonary nodules surrounded with ground glass attenuations and a pleural effusion were seen on the chest computed tomography scan in the presence of an abnormal urinary sediment. An analogous differential problem was described before. ${ }^{31}$ Moreover, in some cases, the diagnosis has been established only after death. ${ }^{3} 1218$

In our present study, four patients had concomitant infections related to their immunosuppressive state, as was largely reported in previous studies. ${ }^{18}{ }^{21}{ }^{23}$ Almost one third of the patients with bacteraemic nocardiosis had a positive blood culture for other pathogens, most notably Gram negative bacteria. ${ }^{17}$ Cytomegalovirus was the most frequent viral copathogen, and was even found to be associated with poor prognosis in patients with cancer and nocardiosis. ${ }^{18}$ This shows that Nocardia spp should not be considered only as a colonising microorganism, especially in severely immunocompromised patients.

Most of the isolates in our study were $N$ asteroides. This predominance has been reported in other European and nonEuropean studies. ${ }^{3461216172132}$ However, the prevalence of $N$ farcinica in our present study was lower than in certain European series (Germany, 60\%; France, 24\%; Italy, $19 \%$ ), ${ }^{27}{ }^{4}$ or in certain patient groups (chronic granulomatous disease, $14 \%$; lung transplant recipients, $30 \%$; patients with nocardaemia, 14.3\%). ${ }^{14} 17$ Usually, high antibiotic resistance of $N$ farcinica, including third generation cephalosporins, emphasises the importance of its identification. ${ }^{27}$

Because the incubation time of the cultures must be prolonged and decontamination techniques adequately selected, the microbiology laboratory should be informed when nocardiosis is clinically suspected. The observation of thin Gram positive, irregularly stained or beaded branching filaments is important in the recognition of Nocardia spp. ${ }^{1}$ In our present study, nocardia were suspected after Gram stain examination in $65 \%$ of specimens from which Nocardia spp could be cultured. Therefore, direct microscopy may provide rapid and useful information that could influences the choice of initial antimicrobial treatment. However, multiple clinical 


\section{Take home messages}

- Nocardiosis mainly affects immunocompromised patients and can become a severe infection

- Differential diagnosis, especially with tuberculosis, often delays the time to diagnosis, which worsens the outcome

- New diagnostic tools, such as the polymerase chain reaction, could provide more rapid and reliable results

- Trimethoprim/sulfamethoxazole was the most commonly prescribed treatment, although it had to be changed in a considerable proportion of our patients because of side effects, and its oral form was not sufficiently efficient in patients with severe infection

- Imipenem should be used as an alternative treatment for severely ill patients, and the sulfa combination for less severe infections

specimens must be examined; in other reports microscopy and cultures were simultaneously positive in only one third of cases. ${ }^{1}$

All $N$ asteroides sensu stricto and $N$ asteroides complex strains identified in our study were susceptible to imipenem, amikacin, TMP/SMX, and ceftriaxone. A sulfonamide containing regimen, particularly TMP/SMX, was the most commonly administered treatment in our study, and is still considered as the treatment of choice. ${ }^{16} 172021$ However, for a considerable number of our patients, TMP/SMX had to be switched to an alternative treatment because of side effects or a lack of efficacy. Moreover, in four patients receiving oral treatment, the treatment was considered to be a therapeutical failure, and two of these patients died. In vitro and animal model data showed that imipenem and amikacin were the most effective anti-nocardial agents and were superior to TMP/SMX. ${ }^{33-35}$ In this study, imipenem alone, or in combination with amikacin proved more effective. Because of its higher bactericidal activity, most authors now propose imipenem monotherapy, or imipenem in combination with amikacin, particularly in severely immunocompromised patients, and in cases of CNS, disseminated, or advanced infection. ${ }^{4612161832}$ As shown recently, linezolid is another potential second line agent. Because of its high activity against all clinically important Nocardia spp, ${ }^{36}$ excellent bioavailability, and positive pilot clinical results, this drug may be particularly useful for patients already undergoing complex medical regimens, such as HIV infected patients or organ transplant recipients. ${ }^{37}{ }^{38}$ However, the longterm toxicity of linezolid still needs to be determined.

The optimal duration of treatment is unknown. Mostly, authors recommend a prolonged course of medication because of the relapsing nature of the infection. In our present study, the median duration of treatment in surviving patients was 90 days. All our patients with the CNS and the cutaneous forms of nocardiosis received a combined medicalsurgical treatment, with a satisfactory clinical outcome.

The observed mortality rate (15\%) in our study was relatively low. In certain studies concerning the general population, ${ }^{41}$ patients with cancer, $^{18}$ organ transplant recipients, ${ }^{15} 1920$ HIV infected patients, ${ }^{2122}$ and patients presenting with bacteraemia, ${ }^{17}$ the observed mortality rate was higher, ranging from $26 \%$ to $63 \%$. The three patients who died in our present study had severe underlying diseases, and the infection was diagnosed late or even after death. Most authors have suggested that mortality is related to the severity of the underlying disease, late diagnosis, and an advanced or disseminated form of nocardial infection. ${ }^{11} 172021$ The clinical course of two patients in our present study was very complicated. One patient showed a metastatic spread to the CNS. The second patient with chronic granulomatous disease experienced the primary infection during TMP/SMX prophylaxis and had several relapses when receiving TMP/ SMX treatment. This clinical case supports a previous suggestion that neither prophylaxis with interferon $\gamma$ nor prophylaxis with a sulfonamide prevents nocardial infection, but may protect against dissemination. Most patients in our study were treated with at least two parenteral antibiotics, including a sulfonamide, and showed good resolution. ${ }^{14}$

"The three patients who died in our present study had severe underlying diseases, and the infection was diagnosed late or even after death"

In conclusion, nocardiosis may become a severe infection and mainly affects profoundly immunocompromised patients. Differential diagnosis, especially with tuberculosis, often delays the time to diagnosis, which worsens the outcome. New diagnostic tools, such as the polymerase chain reaction, could provide more rapid and reliable results. TMT/ SMX was the most commonly prescribed treatment; however in a considerable proportion of our patients it had to be changed for another treatment because of side effects, and its oral form was not sufficiently efficient in patients with severe infection. Imipenem should be used as an alternative treatment for severely ill patients, and the sulfa combination for less severe infections.

\section{ACKNOWLEDGEMENTS}

We thank K Bouchuiguir-Wafa for microbiology laboratory assistance.

\section{Authors' affiliations}

R Matulionyte, I Uçkay, D Lew, J Garbino, Division of Infectious Diseases, Department of Internal Medicine, University of Geneva Hospitals, 1211 Geneva, Switzerland

P Rohner, Clinical Microbiology Laboratory, Division of Infectious Diseases, University of Geneva Hospitals

\section{REFERENCES}

1 Lerner PI. Nocardiosis. Clin Infect Dis 1996;22:891-905.

2 Beaman BL, Beaman L. Nocardia species: host-parasite relationships. Clin Microbiol Rev 1994;7:213-64.

3 Beaman BL, Burnside J, Edwards B, et al. Nocardial infections in the United States, 1972-1974. J Infect Dis 1976;134:286-9.

4 Boiron P, Provost F, Chevrier G, et al. Review of nocardial infection in France 1987 to 1990. Eur J Clin Microbiol Infect Dis 1992;11:709-14.

5 McNeil MM, Brown JM. The medically important aerobic actinomycetes: epidemiology and microbiology. Clin Microbiol Rev 1994;7:357-417.

6 Farina C, Boiron P, Ferrari I, et al. Report of human nocardiosis in Italy between 1993 and 1997. Eur J Epidemiol 2001;17:1019-22.

7 Pintado V, Gomez-Mampaso E, Cobo J, et al. Nocardial infection in patients infected with human immunodeficiency virus. Clin Microbiol Infect 2003;9:716-20.

8 Brown JM, McNeil MM. Nocardia, Rhodococcus, Gordonia, Actinomodura, Streptomyces, and other aerobic actinomycetes. In: Murray PR, Baron EJ, Jorgensen JM, eds. Manual of clinical microbiology. Washington DC American Society for Microbiology, 2003:502-31.

9 Bosshard PP, Abels S, Zbinden R, et al. Ribosomal DNA sequencing for identification of aerobic Gram-positive rods in the clinical laboratory (an 18month evaluation). J Clin Microbiol 2003;41:4134-40.

10 Drancourt $M$, Bollet $C$, Carlioz A, et al. 16S ribosomal DNA sequence analysis of a large collection of environmental and clinical unidentifiable bacterial isolates. J Clin Microbiol 2000;38:3623-30.

11 Pintado V, Gomez-Mampaso E, Fortun J, et al. Infection with Nocardia species: clinical spectrum of disease and species distribution in Madrid, Spain, 1978-2001. Infection 2002;30:338-40.

12 Farina C, Boiron P, Goglio A, et al. Human nocardiosis in Northern Italy from 1982 to 1992. Scand J Infect Dis 1995;27:23-7.

13 Van Burik JA, Hackman RC, Nadeem SQ, et al. Nocardiosis after bone marrow transplantation: a retrospective study. Clin Infect Dis 1997;24:1154-60. 
14 Dorman SE, Guide SV, Conville PS, et al. Nocardia infection in chronic granulomatous disease. Clin Infect Dis 2002;35:390-4.

15 John GT, Shankar V, Abraham AM, et al. Nocardiosis in tropical renal transplant recipients. Clin Transplant 2002;16:285-9.

16 Mari B, Monton C, Mariscal D, et al. Pulmonary nocardiosis: clinical experience in ten cases. Respiration 2001;68:382-8.

17 Kontoyiannis DP, Ruoff K, Hooper DC. Nocardia bacteremia: report of 4 cases and review of the literature. Medicine (Baltimore) 1998;77:255-67.

18 Torres HA, Reddy BT, Raad II, et al. Nocardiosis in cancer patients. Medicine (Baltimore) 2002:81:388-97.

19 Husain S, McCurry K, Dauber J, et al. Nocardia infection in lung transplant recipients. J Heart Lung Transplant 2001 ;21:354-9.

20 Wilson JP, Turner HR, Kirchner KA, et al. Nocardial infection in renal transplant recipients. Medicine (Baltimore) 1989:68:38-57.

21 Uttamchandani RB, Daikos GL, Reyes RR, et al. Nocardiosis in 30 patients with advanced human immunodeficiency virus infection: clinical features and outcome. Clin Infect Dis 1994;18:348-53.

22 Tripodi MF, Adinolfi LE, Andreana A, et al. Treatment of pulmonary nocardiosis in heart-transplant patients: importance of susceptibility studies. Clin Transplant 2001;15:415-20.

23 Javaly K, Horowitz HW, Wormser GP. Nocardiosis in patients with human immunodeficiency virus infection. Medicine (Baltimore) 1992;71:128-38.

24 Lumb R, Greville H, Martin J, et al. Nocardia asteroides isolated from patients with cystic fibrosis. Eur J Clin Microbiol Infect Dis 2002;21:230-3.

25 Loeffler JM, Bodmer T, Zimmerli W, et al. Nocardial brain abscess: observation of treatment strategies and outcome in Switzerland from 1992 to 1999. Infection 2001;29:337-41.

26 Lee GYF, Daniel RT, Brophy BP, et al. Surgical treatment of nocardial brain abscesses. Neurosurgery 2002;51:668-72.

27 Torres $\mathrm{OH}$, Domingo P, Pericas R, et al. Infection caused by Nocardia farcinica: case report and review. Eur J Clin Microbiol Infect Dis 2000;19:205-12.
28 Greenberg $\mathbf{A E}$, Lucas $\mathrm{S}$, Tossou $\mathrm{O}$, et al. Autopsy-proven causes of death in HIV-infected patients treated for tuberculosis in Abidjan, Côte d'lvoire. AIDS 1995;9:1251-4.

29 Subhash HS, Christopher DJ, Roy A, et al. Pulmonary nocardiosis in human immunodeficiency virus infection: a tuberculosis mimic. J Postgrad Med 2001;47:30-2

30 Olson ES, Simpson AJH, Norton AJ, et al. Not everything acid fast is Mycobacterium tuberculosis - a case report. J Clin Pathol 1998:51:535-6.

31 Gibb W, Williams A. Nocardiosis mimicking Wegener's granulomatosis. Scand J Infect Dis 1986; 18:583-5

32 Hui CH, Au VWK, Rowland K, et al. Pulmonary nocardiosis re-visited: experience of 35 patients at diagnosis. Respir Med 2003:97:709-17.

33 Gombert ME, Aulicino TM, duBouchet L, et al. Susceptibility of Nocardia asteroides to new quinolones and beta-lactams. Antimicrob Agents Chemother 1987;31:2013-14.

34 Gombert ME, Berkowitz LB, Aulicino TM, et al. Therapy of pulmonary nocardiosis in immunocompromised mice. Antimicrob Agents Chemother 1990;34:1766-8.

35 Gombert ME, Aulicino TM, duBouchet L, et al. Therapy of experimental cerebral nocardiosis with imipenem, amikacin, trimethoprimsulfamethoxazole, and minocycline. Antimicrob Agents Chemother 1986;30:270-3.

36 Brown-Elliott BA, Ward SC, Crist CJ, et al. In vitro activities of linezolid against multiple Nocardia species. Antimicrob Agents Chemother 2001;45:1295-7.

37 Moylett EH, Pacheco SE, Brown-Elliott BA, et al. Clinical experience with linezolid for the treatment of Nocardia infection. Clin Infect Dis 2003;36:313-8.

38 Lewis KE, Ebden P, Wooster SL, et al. Multi-system infection with Nocardia farcinica-therapy with linezolid and minocycline. J Infect 2003;43:199-202 